\title{
A Service Discipline for Support of IP QoS in IEEE802.11 Networks
}

\author{
António Grilo, Mário Macedo and Mário Nunes \\ INESC, R. Alves Redol, No9, 1000 Lisboa, PORTUGAL
}

Email:amg@cris.inesr.pt

Key words: $\quad 802.11$, IP QoS, Scheduling Discipline, Discrete Event Simulation.

Abstract: $\quad$ This paper presents a QoS aware service discipline based on estimated transmission times for use in IEEE 802.11 networks. This algorithm works based on the flow specifications defined by the IETF for the IP QoS reference models, coupling layer 2 and layer 3 QoS. Simulation results show that the proposed service discipline performs better than Weighted Round Robin in IEEE $802.11 \mathrm{~b}$ networks, providing a better optimisation of network resources while fulfilling the QoS requirements of real-time services such as packet telephony and videoconference.

\section{INTRODUCTION}

Wireless LANs (WLANs) are becoming a major growth factor for the wireless networks industry, promising to replace most wired LAN infrastructures in the near future. The main advantage of WLANs over their wired counterparts is the increased mobility, allowing users to roam inside their enterprise or campus without interrupting their communication sessions. The mostly commercialised WLAN products are nowadays based on the IEEE 802.11 standard [1]. The physical layer was firstly designed to work in the $2.4 \mathrm{GHz}$ frequency band. Starting with a bit rate of $2 \mathrm{Mbps}$, it reached $11 \mathrm{Mbps}$ with $802.1 \mathrm{lb}$. This will be followed by 802.1la, which works in the $5 \mathrm{GHz}$ band and is able to provide bit rates as high as $54 \mathrm{Mbps}$. 
Simultaneously with the growth of wireless communications there is the trend for multimedia communications over IP with Quality of Service (QoS) support. The use of multimedia communication services such as telephony, videoconference and streamed video over IP is now a reality in corporate networks and promises to expand to the global Internet. This has prompted the IETF to develop the IntServ and DiffServ reference models for QoS support in IP networks.

This paper addresses both issues, presenting a solution for QoS support in 802.11 networks ${ }^{4}$. The proposed solution can be easily adapted to other WLAN technologies. The paper is focused on a scenario of wireless access to a high performance IP core network, as can be found in corporate or university environments. In order to support end-to-end QoS and avoid traffic bottlenecks at the radio access interface, layer 3 and layer 2 QoS must be closely coupled. This is achieved by a QoS aware scheduling algorithm that works on top of the current 802.11 MAC layer and whose input consists of IP QoS parameters. Performance evaluation is attained through software simulation and considers $802.11 \mathrm{~b}$, which is now widely accepted.

\section{THE IEEE802.11 MAC}

The QoS support mechanisms defined for the MAC layer of IEEE 802.11 are quite basic. The standard defines only two priority values for a data packet: Contention and ContentionFree. The former specifies that data is to be transmitted by means of the Distributed Coordination Function (DCF), which uses a CSMA/CA mechanism. The latter specifies data transmission during the Contention Free Periods (CFPs) using a polling mechanism controlled by the Point Coordination Function (PCF) at the Access Point (AP). Within a cell, DCF and PCF modes are time multiplexed under the control of the AP (see Figure 1). A superframe is formed by a CFP followed by a contention period.

${ }^{4}$ The work presented is partially funded by the European Union in the framework of the IST program project MOICANE. The authors alone are responsible for the content of the paper. 


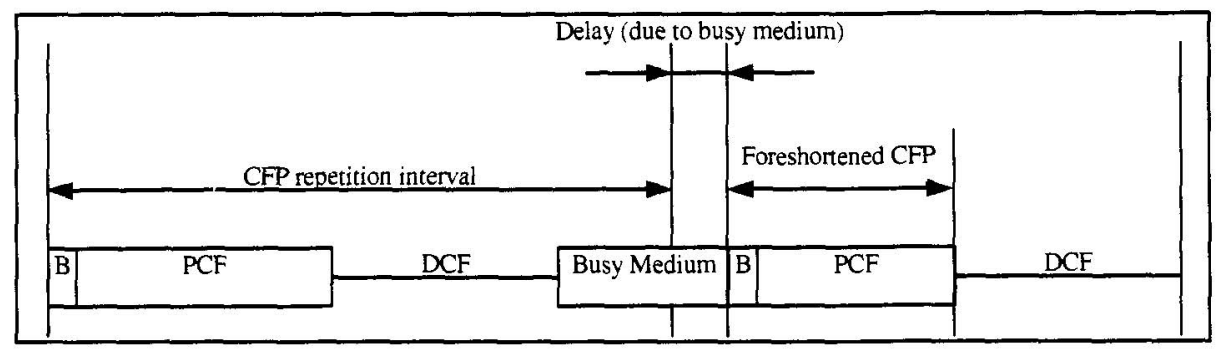

Figure 1. Beacons and contention free periods

During the time interval between two CFPs, the WLAN works in DCF mode using CSMA/CA for transmission. If the end of the DCF occurs during the transmission of a packet, the start of the next CFP is delayed until the packet source ends transmission (this may only happen after all fragments of the packet are successively transmitted or the dwell time boundary expires). Additionally, the CFP is shortened in order to finish at the due time (this is illustrated by the second CFP in Figure 10). These delays and lack of preemptive control present an obvious difficulty for the handling of real-time traffic. For this reason, the dwell time boundary of the DCF should be carefully selected. On the other hand, the DCF mode cannot be eliminated because registration of terminals with the PCF takes place in DCF mode.

Packet priorities are implemented by defining 3 IFSs (Interframe Spaces) of different lengths:

- SIFS (Short IFS): This is the shortest IFS. It is used for transmission of high priority packets: Acknowledgements of data packet fragments, CTS frames, PCF DATA frames and DCF DATA frames (except the first fragment of a MAC SDU).

- PIFS (PCF IFS): This is greater than SIFS. After this interval expires, any PCF mode frames can be transmitted.

- DIFS (DCF IFS): This is greater than PIFS. After this interval expires, any DCF mode frames can be transmitted asynchronously according to the backoff mechanism (see below).

The DCF mode is based on a CSMA/CA mechanism. The access control scheme is shown in Figure 2. 


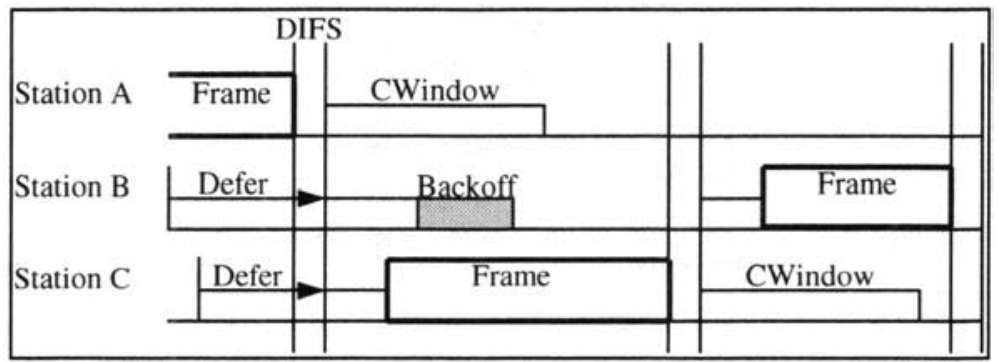

Figure 2. Backoff mechanism in DCF

A station that intends to transmit and senses the channel busy will wait for the end of the ongoing transmission, then wait for a time period of DIFS length, and then randomly selects a time slot within the backoff window. If no other station started transmitting before this slot is reached (i.e. another station that selected an earlier slot) it starts the transmission of a fragment with maximum size aFragmentationThreshold. Collisions can now only occur in the case that two stations have selected the same slot. If another station has selected an earlier slot, the station freezes its backoff counter, waits for the end of this transmission and now only waits for the slots remaining from the previous competition. After the successful transmission of the first fragment of an MSDU, the remaining fragments are transmitted is succession separated by a SIFS interval. Transmission ends when all fragments of an MAC SDU (MSDU) are transmitted or the maximum dwell time expires.

In order to guarantee undisturbed transmission even if hidden terminals are present, an RTS/CTS mechanism is used. When this mechanism is applied, the contention winner does not transmit the data immediately. Instead it sends an RTS frame to which the receiver answers with a CTS frame. This guarantees that all stations in the range of either the sender or the receiver know that a packet will be transmitted. Only then the sender transmits the data frames. While the two extra messages present additional overhead, the mechanism is specially useful in the case of large data packets.

The PCF mode is based on a polling mechanism controlled by the AP as depicted in Figure 3. 


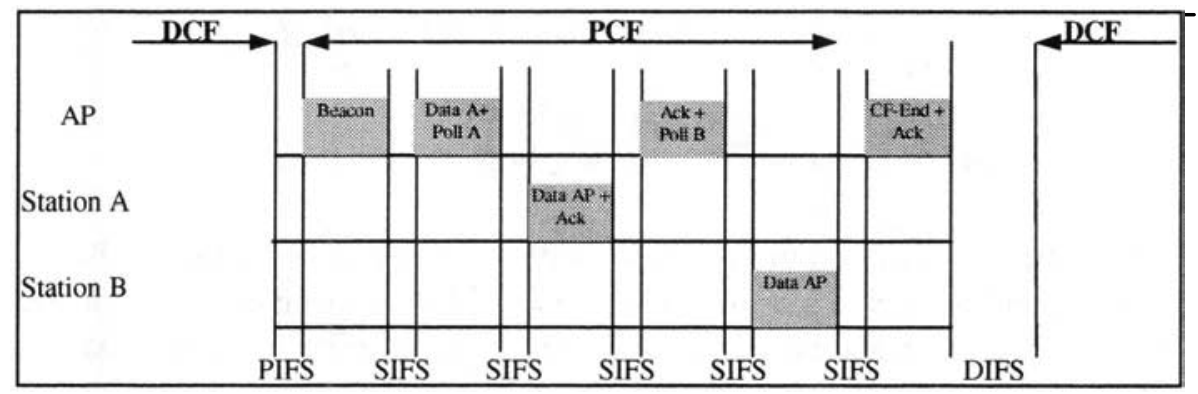

Figure 3. Polling mechanism in PCF

During the CFP, the AP polls the stations registered on its polling list and allows them undisturbed contention free access to the medium. As already said, in order to get on the polling list the STAs have to apply once during the DCF period. The maximum duration of a CFP is given by the 802.11 Management Information Base (MIB) variable aCFPMaxDuration.

During the CFP a frame can be a composite of control and data information. As such, the following combinations are allowed for PCF frames: DATA; CF-ACK, CF-POLL, DATA+CF-ACK; DATA+CFACK + CF-POLL and CF-ACK +CF-POLL. Only the AP has the capability to issue frames with CF-POLL. The STAs can only answer with DATA, CF$\mathrm{ACK}$ or DATA $+\mathrm{CF}-\mathrm{ACK}$. Each polling request must be answered with the maximum of 1 data fragment of maximum size aFragmentation Threshold. If the STA does not answer a polling request within an interval of PIFS, the AP concludes that the STA has nothing to transmit and resumes CFP by sending downlink data and/or polling another STA.

It is useful to evaluate the maximum throughput that can be achieved with the PCF. Considering that all fragments have the same size, the maximum throughput achieved by the PCF mode can be approximated by the following expression:

$$
\text { MaxThroughput }=\frac{\text { aCFPMaxDuration }}{\text { superframe_duration }} \times \frac{\text { frgment_size }}{\text { fragment_time }}
$$

The average fragment transmission time is then the average between uplink and downlink fragment transmission times. As already seen, the transmission of a fragment in the uplink direction involves the transmission of a CF_POLL frame, followed by a DATA frame and finally a CF_ACK frame, while in the downlink direction no polling frame is needed. As such, the average fragment transmission time can be calculated as follows, considering piggyback of acknowledgements for uplink traffic: 


$$
\begin{aligned}
& \overline{\text { fragment_time }}=\frac{\text { Uplink_load } \times C F_{-} P O L L_{-} \text {time }}{\text { Total_load }} \\
& + \text { DATA_time }+C F_{-} A_{\text {ACK_time }}
\end{aligned}
$$

The transmission of a frame involves the transmission of a physical layer preamble and header at the lowest bit rate $(1 \mathrm{Mbps})$, followed by the MAC PDU (MPDU) transmitted at the PSDU bit rate. As such, the time taken to transmit a CF_POLL frame is the following.

$$
C F_{-} P O L L_{-} \text {time }=\frac{P L C P_{-} \text {overhead_size }}{P L C P_{-} \text {header } \text { bitrate }_{\text {e }}}+\frac{C F_{-} P O L L_{-} \text {size }}{P S D U_{-} \text {bit } \text { rate }}+S I F S
$$

The time taken to transmit a CF-ACK frame is calculated in a similar way:

$$
C F \_A C K_{-} \text {time }=\frac{P L C P_{-} \text {overhead_size }}{P L C P_{-} \text {header_bitrate }}+\frac{C F_{-} A C K_{-} \text {size }}{P S D U_{-} \text {bit_rate }}+S I F S
$$

The time taken to transmit a DATA frame is calculated as follows:

$$
\begin{aligned}
& C F_{-} D A T A_{-} t i m e=\frac{P L C P_{-} \text {overhead_size }}{P L C P_{-} \text {header_bitrate }} \\
& +\frac{D A T A_{-} h e a d e r_{-} \text {size }+ \text { fragment_size }}{P S D U_{-} \text {bit_rate }}+S I F S
\end{aligned}
$$

Both DCF and PCF have advantages that can be exploited in the appropriate context. DCF is the most appropriate in a context where there is no network infrastructure and users must form temporary ad hoc networks to communicate directly between each other. The literature provides some interesting publications on the evaluation [2] and improvement [3] [4] [5] of the 802.11 CSMA/CA algorithm.

On the other hand, if the objective is to offer a permanent network infrastructure to provide access to the Intranet/Internet with guaranteed QoS bounds, PCF is the best choice. This is the scenario considered in the present paper. 


\section{IP QOS PARAMETERS}

The IETF has defined two main models for QoS support in IP networks: Integrated Services (IntServ) and Differentiated Services (DiffServ).

IntServ was created to provide end-to-end QoS. The Resource Reservation Protocol RSVP [6] protocol is used for signalling QoS requests from the application to the network. In this model, the originator endpoint uses the RSVP PATH message to advertise the bandwidth requirements of a flow. That message traverses the network routers until it reaches the destination endpoint. The destination compares the required resources with the available resources and answers with a RSVP RESV message. This message travels all the way back making resource reservation for the flow at each traversed router. The IntServ model specifies three main service classes: Guaranteed Service (GS), Controlled Load (CL) and Best Effort (BE). The GS class presents the highest level of QoS guarantee, while the $\mathrm{BE}$ calss presents the lowest level.

Due to the scalability problems of IntServ, the IETF developed a simpler QoS model called DiffServ [7]. In this model, user flows are only controlled at the edge of network domains, being classified in one of several classes. Within a network domain, all flows that belong to the same class are aggregated and forwarded with the same priority. Until now the IETF has identified three main traffic classes or per-hop behaviours (PHB): Expedited Forwarding (EF), Assured Forwarding (AF) and Best Effort (BE). These PHBs correspond roughly to the service classes defined for IntServ. The EF class is to be used for high priority services with tight QoS bounds, such as packet telephony, its main application being the support of virtual leased lines. The AF class has lower priority than EF, but it is still able to provide some QoS guarantees. Finally, the BE class is suitable for less demanding services, using the resources left by EF and AF.

The IP QoS parameters for a flow form a flow specification (FlowSpec) [8]. FlowSpecs are used in both IntServ and DiffServ.

In a FlowSpec, the transmission rate is parameterised as a token bucket. A token bucket has a maximum volume (Token Bucket Size) and continuously fills in at a certain byte rate (Token Rate), which corresponds to the average transmission rate. If the bucket becomes full, the incoming tokens will be thrown away. If the token bucket contains sufficient credit, the application may send data; reducing the available credit by that amount. If sufficient credits are not available, the application must wait or discard the extra traffic. If an application has been sending at a low rate for a period of time, it clearly may send a large burst of data at once with a rate not higher than Peak Rate until it runs out of credit. Having done so, it must limit itself to sending at Token Rate until its data burst is exhausted. In constant rate 
applications, the Token Rate is equal to the Peak Rate, and the Token Bucket Size is chosen to accommodate small variations.

Another important FlowSpec parameter is the Maximum Transmission Unit (MTU), which is the maximum packet size, in bytes, generated by the host.

Transmission delay is also important. While some applications such as Web access are not very sensitive to delay, real-time services such as telephony demand minimum delay in order to achieve acceptable quality. The Maximum Delay of a service is the maximum acceptable delay between transmission of a bit by the sender and its reception by the intended receiver(s), usually expressed in microseconds. If a receiving application requires data to be delivered in the same pattern that the data was transmitted, it may be necessary for the receiving host to briefly buffer data as it is received so that the receiver can restore the old transmission pattern. An easy example of this is a case where an application wishes to transmit data such as voice samples, which are generated and played at regular intervals. The regular intervals may be distorted by queuing effects in the network and the receiver may have to restore the regular spacing. The amount of buffer space that the receiving host is willing to provide determines the Maximum Delay Variation (delay variation is also known as jitter) permitted for individual packets within a given flow.

The Loss Sensitivity parameter states how sensitive the flow is to losses. The Loss Sensitivity is defined as the number of MTU-sized packets that may be lost out of the number of MTU-sized packets specified in the Loss Interval parameter. Another useful parameter is the Burst Loss Sensitivity, which states how sensitive the flow is to losses of consecutive packets. It is defined as the maximum number of consecutive MTU-sized packets that may be lost.

Finally, the Quality of Guarantee specifies the level of commitment of the network towards the QoS parameters. As we have already seen, this corresponds to the service classes of IntServ and DiffServ. Throughout the rest of this paper, the DiffServ service classes will be used to characterise this parameter.

\section{SCHEDULING IN 802.11}

Among the common service disciplines that could be used to implement the IEEE 802.11 PCF, we have identified two main families: Weighted Fair Queuing (WFQ), and Weighted Round Robin (WRR) [9]. 
WRR was chosen for performance benchmarking because it is widely accepted and simple to adapt to a WLAN environment, as it needs no information about the queued messages at the STAs or AP.

On the other hand, WFQ is difficult to adapt to a polling mechanism like the IEEE 802.11 PCF, since there is no way for the AP to know neither about the presence of queued packets at the terminals nor the packet generation times. The same difficulties apply to other WFQ family disciplines, such as $\mathrm{W} 2 \mathrm{FQ}$, and $\mathrm{W} 2 \mathrm{FQ}+$. Nevertheless, it is possible to estimate the presence of packets on the terminal's queues, and therefore implement a version of WFQ based on packet presence expectations if we observe the leaky bucket's state. If the leaky bucket is empty, it is more probable that the corresponding terminal has already one packet to transmit than if the leaky bucket is full. However, this procedure is not accurate, because it only indicates a probability of packet generation.

Most packet scheduling disciplines are not specially designed to meet QOS constraints such as those of IP QoS models. For example, RFC 2598 [10] suggests the use of simple priority for EF traffic on the DiffServ routers, as an alternative to simple WRR, in order to implement a low delay, and a low jitter service for the EF.

\subsection{Weighted Round Robin}

The Weighted Round Robin (WRR) service discipline can be easily adapted to the IEEE802.11 environment. The algorithm generates a polling rate proportional to the weight of the flows, but it also tries to distribute the polls as uniformly as possible. The algorithm has similarities with the Deficit Weighted Round Robin algorithm [9].

Let $\mathrm{FR}_{\mathrm{avi}}$ be the average fragment rate of flow $i$, while $L F_{\mathrm{i}}$ is the average fragment length. The weight of each flow $i, \Phi_{i}$ is calculated by the expression:

$$
\phi_{\mathrm{i}}=\frac{F R_{\mathrm{avi}}}{\sum_{\mathrm{i}} F R_{\mathrm{avi}}}
$$

Let $C_{i}$ be the transmission credit of flow i. The WRR algorithm can be implementated as follows:

1. Init all $C_{i}=0$;

2. Choose $m$ such that $C_{m}=\max (C i)$. If there are many equals, choose the first.

3. Send a poll to flow $m$; 
4. Decrease the credits of flow $m$ making $C_{m}=C_{m}-1$ and distribute those credits among all flows making $C_{\mathrm{i}}=C_{\mathrm{i}}+\phi_{\mathrm{i}}$;

5. Go to step 2 .

Note that this algorithm can only work with acceptable performance when the low level traffic characteristics of the sources are well known. In practice $F R_{\text {avi }}$ and $L F_{\mathrm{i}}$ are not known in advance as these parameters dependent on layer 2 .

\subsection{Scheduling based on Estimated Transmission Times}

The service discipline proposed in this paper bases its scheduling decisions on the estimated packet transmission times (SETT - Scheduling based on Estimated Transmission Times). A similar procedure was used for the rt-VBR traffic scheduling in IEEE802.14 networks [11].

A variable $t_{\text {poll }}$ is associated to each flow, representing the estimated transmission time of a burst from the source, and therefore the more or less ideal time for the AP to issue a poll to the source. $T_{\text {polling }}$ is a variable that represents the time interval between polls to be issued to that flow.

SETT issues a poll to the flow that has the minimum $\mathrm{t}_{\text {poll }}<=\mathrm{t}$, where $\mathrm{t}$ is the real time clock of the system. Each time a station is polled, the next $t_{\text {poll }}$ is calculated as $\mathrm{t}_{\text {poll }}=\mathrm{t}_{\text {poll }}+\mathrm{T}_{\text {polling. }}$.

When a flow is bursty, those polls are used to detect the beginning of the burst. After the detection, SETT enters a transmission procedure where the flow is polled at the maximum possible rate, not higher than the Peak Rate.

The burst detection algorithm provides different treatment for each service class:

\section{A. Algorithm for EF flows}

Generally, EF flows have a contracted transmission rate $\mathrm{R}_{\mathrm{av}}$ (Token Rate) and a Token Bucket Size $\sigma$. Other relevant parameters of EF flows are MTU, the Peak Rate and $\mathrm{T}_{\text {dmax }}$ (end-to-end Maximum Delay).

For the EF flows, SETT will try to transmit all the bucket size within a fraction $\alpha$ of $T_{\text {dmax }}$ leaving enough time to be spent throughout the core network and destination access network. Let $L F$ be the length of the longest allowed fragment of a flow. $L F$ is calculated as follows:

$$
L F=\min (M T U, \text { aFragmentationThresh old })
$$

The polling interval $T_{\text {polling }}$ for detecting the beginning of a burst is given by: 


$$
T_{\text {polling }}=\min \left(\frac{L F}{R_{\mathrm{av}}}, \frac{T_{\mathrm{dmax}} \times \alpha}{\min \left(\beta, \frac{\sigma}{L F}\right)}\right)
$$

The polling interval $T_{\text {poling }}$ is the minimum value between the mean interfragment period and the fraction of the detection latency, which is the maximum latency that can be used to detect the burst at the access network.

For constant rate flows, this polling interval implies an average access delay not higher than the average inter-fragment period. Also for this type of flows, this also allows us to comply with the contracted Token Rate for the flow, $R_{\mathrm{av}}$.

If the delay requirements are severe, the detection latency term will result in a lower polling interval. For sources with long bursts (high $\sigma$ ), the algorithm must guarantee that all fragments of the burst are transmitted within the fraction of $T_{\text {dmax }}$ allotted to the access network. As such, the polling interval will depend on the ratio between $\sigma_{i}$ and $L F$, but not higher than parameter $\beta$.

\section{B. Algorithm for AF flows}

Generally, AF flows have a contracted average transmission rate $R_{\mathrm{av}}$ (Token Rate) and a token bucket size $\sigma$. Other relevant parameters of the AF flows are the MTU, and the Peak Rate.

For an AF flow, the algorithm is similar to the EF procedure described above, the only difference being the computation of the polling interval, which now is given by:

$$
T_{\text {polling }}=\frac{L F}{R_{\mathrm{av}}}
$$

This is due to the fact that the delay bounds are not as severe for AF than for EF.

\section{Algorithm for Best-Effort flows}

Best-Effort flows have no QOS requirements. As such, scheduling of BE flows is reduced to a plain Round Robin service discipline, with polls being issued whenever there are no pending polls for the EF or AF service classes. This procedure allows the even distribution of free capacity among the BE 
flows. The AF flows are also included in the Round Robin cycle as they should also benefit from extra bandwidth, as suggested in RFC 2597 [12]. However, the transmission rates for the AF flows can never exceed the specified Peak Rate.

\section{SIMULATION RESULTS}

The SETT and WRR algorithms were simulated using a discrete event simulator developed at INESC. The engine of the simulator was already used in the past to study the performance of the DECT Packet Radio System [13]. The simulator allows the manipulation of some of the parameters that constitute the 802.11 MIB. The parameterisation used in this simulation is shown in Table 1.

Table 1. Simulation parameters 802.11 parameters

ASlotTime $20 \mathrm{~ms}$

$\begin{array}{lc}\text { aFragmentation } & 1024 \text { bytes } \\ \text { Threshold } \\ \text { SIFS } & 20 \mu \mathrm{s} \\ \text { PIFS } & 30 \mu \mathrm{s} \\ \text { DIFS } & 40 \mu \mathrm{s} \\ \text { Beacon Interval } & 100 \mathrm{~ms} \\ \text { DTIM Interval } & 3 \\ \text { aCFPMaxDuration } & 880 \mathrm{~ms} \\ \text { aCFPRate } & 3 \\ \text { PLCP sublayer } & \mathrm{DSSS} \\ \text { PLCP overhead } & 192 \mathrm{bits} \\ \text { PLCP preamble } & 1 \mathrm{Mbps} \\ \text { and } & \\ \text { header bit rate } & \\ \text { PSDU bit rate } & 11 \mathrm{Mbps}(802.11 \mathrm{~b}) \\ \alpha & 0.40 \\ \beta & 6\end{array}$

SETT parameters 


\begin{tabular}{|c|c|c|}
\hline 802.11 parameters & ASlotTime & $20 \mathrm{~ms}$ \\
\hline $\begin{aligned} \text { Physical } & \text { medium } \\
\text { parameters } & \end{aligned}$ & Frame Loss Ratio & $0 \%$ \\
\hline 802.11 parameters & ASlotTime & $20 \mathrm{~ms}$ \\
\hline
\end{tabular}

As can be seen, a CFP occupies $880 \mathrm{~ms}$ (aCFPMaxDuration) of each superframe of $900 \mathrm{~ms}$ (aCFPRate $\times$ DTIM Interval $\times$ Beacon Interval). Such a parameterisation assumes that all data traffic is transmitted during the CFP, relegating the contension period for the exchange of management and control information. It also assumes a scenario of fixed wireless access where mobility is negligible (e.g. within a room). Otherwise a handover delay of $880 \mathrm{~ms}$ would not be admissible. The frame loss ratio due to physical layer errors is $0 \%$, which is not very far from the truth in a corporate environment.

During the simulations we have considered three types of traffic sources: bursty data (e.g. HTTP sessions), VoIP and video.

The model for bursty data sources follows the Source Type 1 defined for 802.14 performance evaluation [14], which consists on a Poisson distribution, where message sizes and respective probabilities are the following: $(64,0.6),(128,0.06),(256,0.04),(512,0.02),(1024,0.25)$ and (15 18,0.03). In our simulations, each busty data source generates $200 \mathrm{Kbps}$.

The audio source model generates new messages with a constant rate and size. We have considered $8 \mathrm{Kbps}$ audio sources (G.729) where 20-byte messages are periodically generated with an interval of $20 \mathrm{~ms}$. We have also considered an RTP/UDP/IP overhead of 40 bytes per message, which results in an overall bit rate of $24 \mathrm{Kbps}$ ( 60 bytes per message).

For the video source model we have considered a frame rate of 15 frames-per-second (fps), with one key-frame (synchronization frame which uses only intraframe compression, such as the $I$ frame in MPEG) being generated once in every 15 frames. Each source generates video at $250 \mathrm{Kbps}$ and each key-frame is 28032 bytes long. The size of the other frames is given by a normal distribution $(\mu, \sigma)=(229,20)$.

In the simulations, each wireless terminal runs only one session and all sessions are bi-directional, i.e. each terminal is the source of uplink data and the sink of downlink data for the session it runs.

Each traffic type is assigned a RFC 1363 flow specification, as provided in Table 2. The SETT algorithm uses only a subset of the FlowSpec parameters. Note that the maximum delay values for audio and video represent end-to-end delay. ITU-T recommendation G. 114 [15] specifies a Maximum Delay of $150 \mathrm{~ms}$ for telephony. As already seen, SETT will try to 
spend no more than $40 \%$ (parameter $\alpha$ ) of that Figure (i.e. $60 \mathrm{~ms}$ ) in the access network in order to leave enough time to be spent in the core $(20 \%)$ and destination access (40\%) networks. Video is considered to be real-time (e.g. videoconference) and as such it presents the same Maximum Delay parameter as VoIP. The tests only address the EF and BE service classes. The AF service class is left for further study.

Table 2. Flow specifications for the several traffic types

\begin{tabular}{lccc}
\hline $\begin{array}{c}\text { QOS } \\
\text { Parameters }\end{array}$ & Data & VoIP & Video \\
\hline $\begin{array}{c}\text { Service } \\
\text { Class } \\
\text { Token Rate }\end{array}$ & BE & EF & EF \\
Token Burst & - & $24 \mathrm{Kbps}$ & $250 \mathrm{Kbps}$ \\
$\begin{array}{c}\text { Size } \\
\text { Peak Rate }\end{array}$ & $120 \mathrm{Bytes}$ & $28032 \mathrm{Bytes}$ \\
MTU & - & $24 \mathrm{Kbps}$ & $11 \mathrm{Mbps}$ \\
$\begin{array}{l}\text { Maximum } \\
\text { Delay }\end{array}$ & - & $60 \mathrm{Bytes}$ & $28032 \mathrm{Bytes}$ \\
$\begin{array}{l}\text { QoS } \\
\text { Parameters }\end{array}$ & - & $150 \mathrm{~ms}$ & $150 \mathrm{~ms}$ \\
\hline
\end{tabular}

The first experiment considers a video session running simultaneously with $n$ VoIP sessions. Figure 4 shows the average packet delay for the VoIP streams (it includes both uplink and downlink values) as $n$ increases. As can be seen, WRR performs better than SETT. Nevertheless, the average packet delay of SETT is kept slightly higher than $10 \mathrm{~ms}$ for less than $16 \mathrm{VoIP}$ sessions, which is acceptable for telephony. Above 17 VoIP sessions, average delay is unacceptable (i.e. above $60 \mathrm{~ms}$ ) for both scheduling disciplines. 


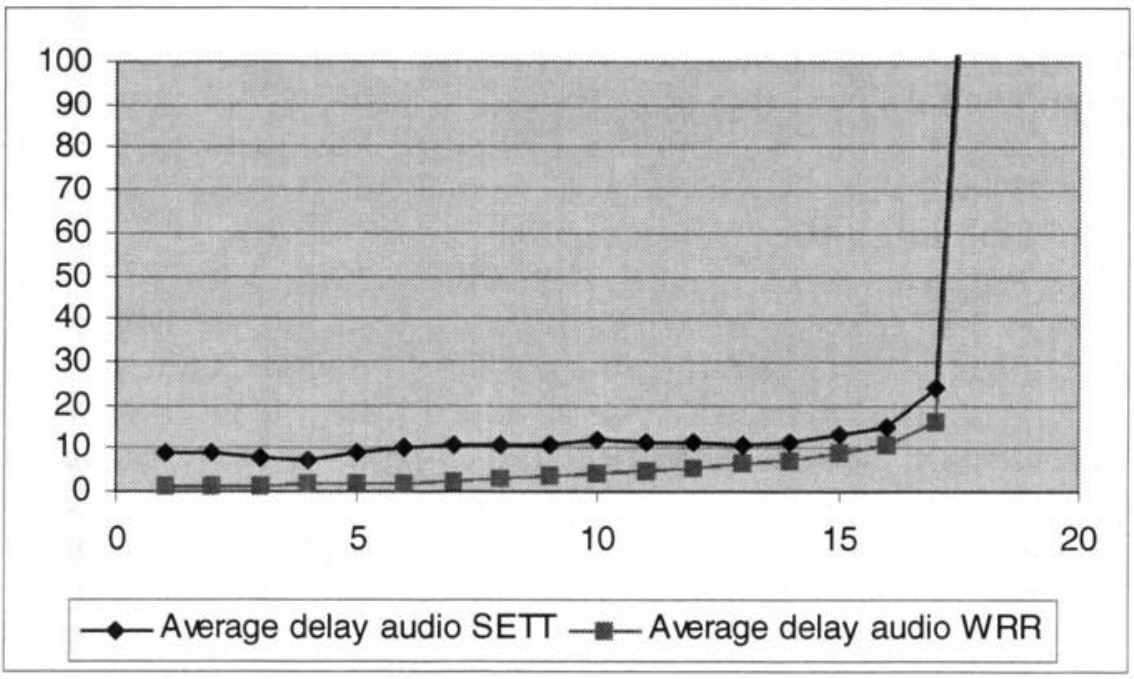

Figure 4. Simulation 1 : Average packet delay (ms) for the VoIP streams.

The maximum packet delay is depicted in Figure 5. It shows that SETT starts to present delay values of more than $60 \mathrm{~ms}$ with more than $15 \mathrm{VoIP}$ sessions. In WRR, maximum packet delay rises more smoothly, going above 60 ms only with more than 17 VoIP sessions.

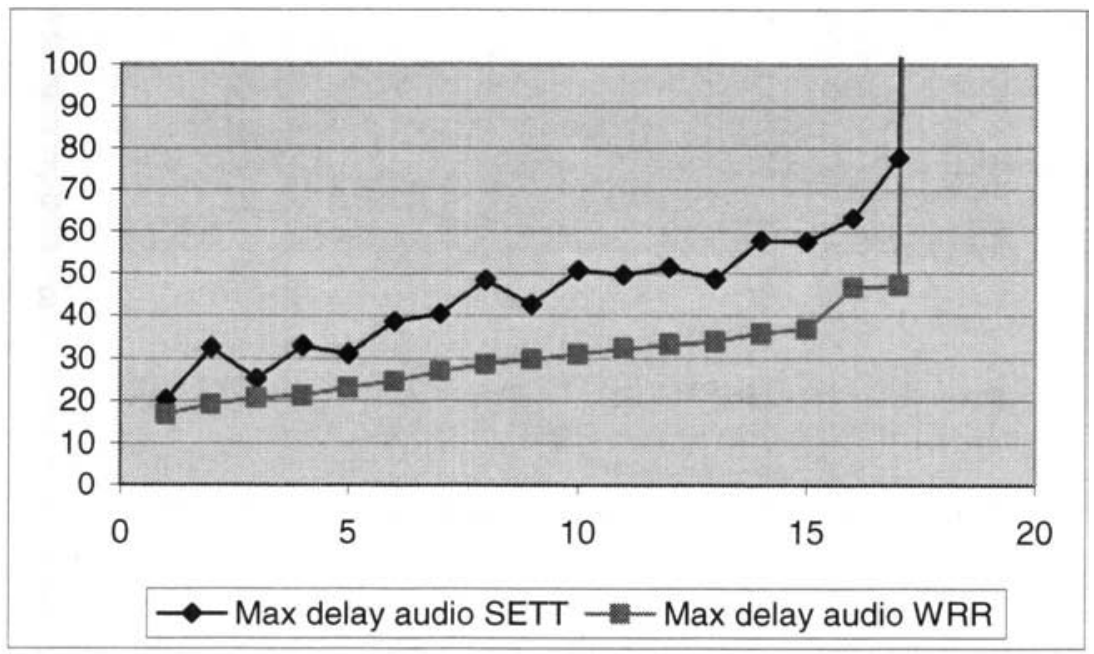

Figure 5. Simulation 1: Maximum packet delay (ms) for the VoIP streams. 
The average packet delay for the video session is depicted in Figure 6 . Although WRR performs slightly better in the beginning, SETT starts to perform better for more than 5 VoIP sessions. Above 8 VoIP sessions, the delay presented by SETT is less than $50 \%$ of the delay presented by WRR. Above 12 VoIP sessions, average delay rises above $60 \mathrm{~ms}$ for WRR, while for SETT this only happens with more than 15 VoIP sessions.

The maximum packet delay is depicted in Figure 7 and shows that maximum delay rises above $60 \mathrm{~ms}$ from the beginning for both service disciples, though SETT performs always better than WRR. This is due to the keyframes of 28032 bytes, which would need $3.7 \mathrm{Mbps}$ to be transmitted in $60 \mathrm{~ms}$. Although the instantaneous physical bit-rate is $11 \mathrm{Mbps}$, this value is impossible to achieve due to the overhead. As such, videoconference transmission over $802.1 \mathrm{lb}$ can be hampered by high delays. A solution is to use the key-frames for decompression of the smaller frames that follow, not showing them to the user. The impact of this technique in the quality perceived by the user must be evaluated. With 802.1 la at bit-rate higher than $20 \mathrm{Mbps}$ this problem will probably cease to exist.

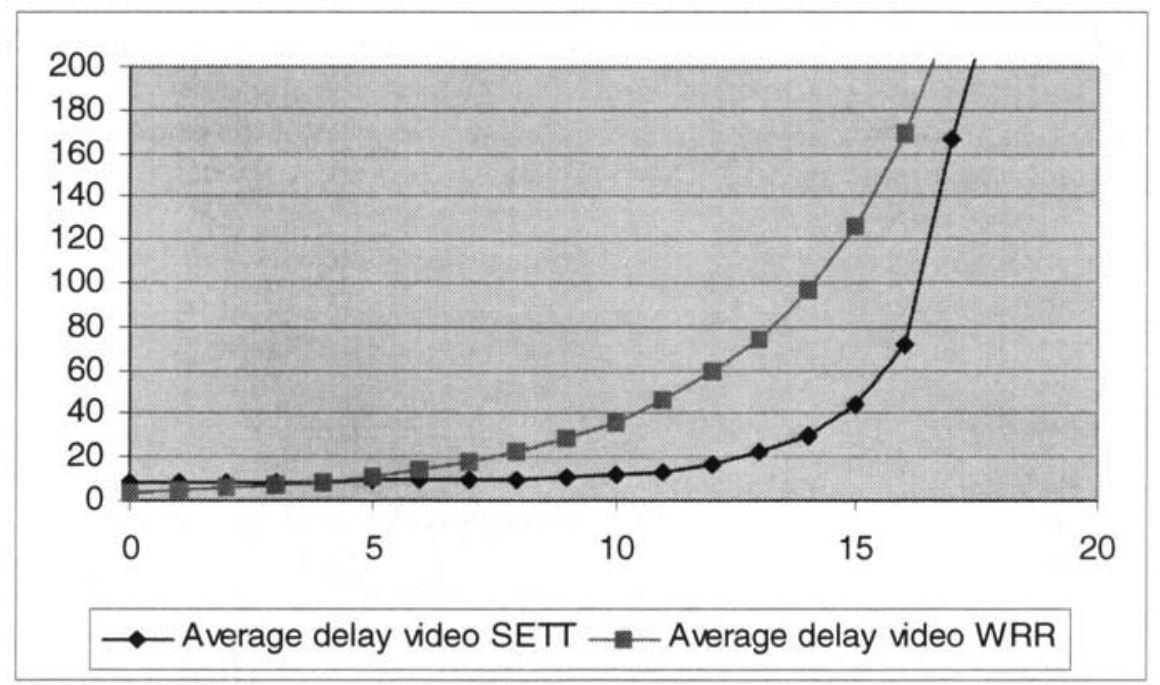

Figure 6. Simulation 1 : Average packet delay (ms) for the video stream 


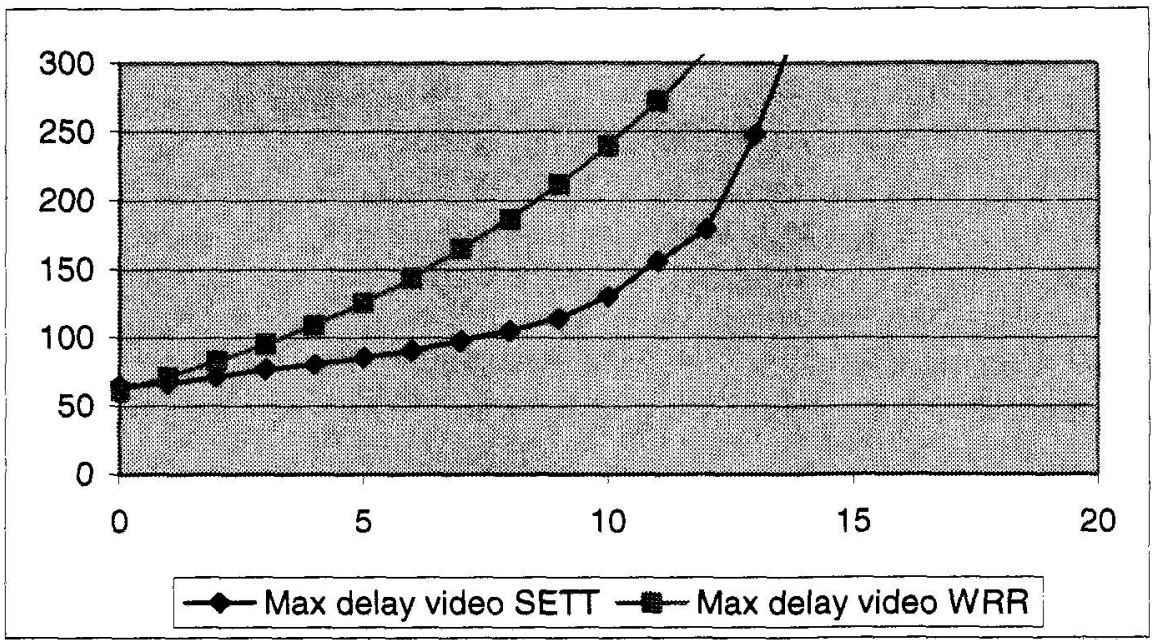

Figure 7. Simulation 1: Maximum packet delay (ms) for the video stream.

The second experiment considers a video session running simultaneously with 2 VolP sessions and $n$ bursty data sessions, with increasing $n$. As delay is not much relevant for BE flows, Figure 8 depicts the throughput instead. This is the sum of uplink and downlink throughput values. As can be seen, the disciplines have similar performance. Throughput starts to rise, achieving a maximum of approximately $3 \mathrm{Mbps}$ with 8 data sessions for SETT. WRR rises a little above that value (3300 Mbps), at the cost of audio and video performance as will be shown.

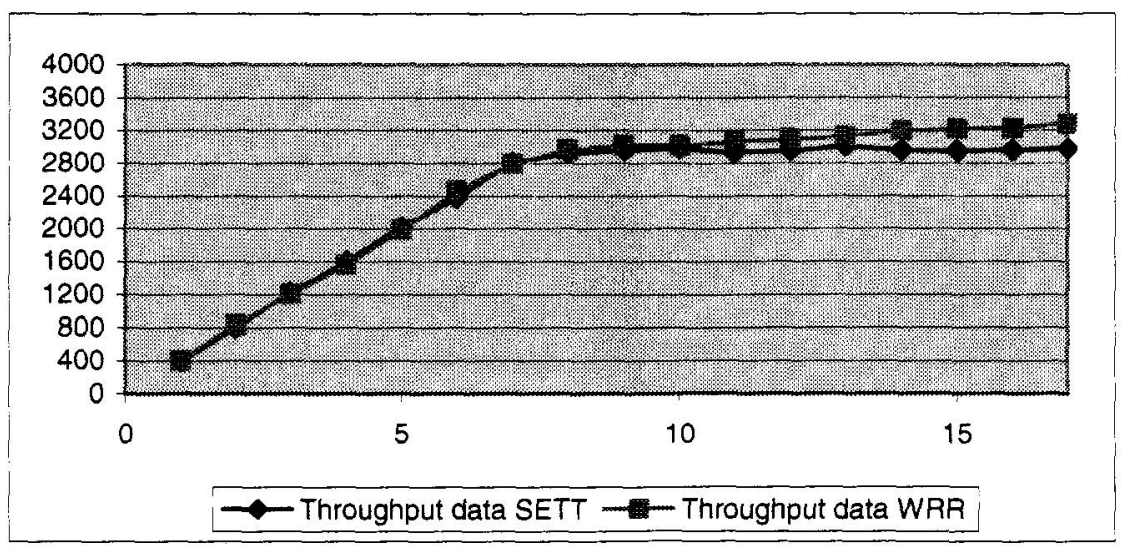

Figure 8. Simulation 2: Throughput (Kbps) of bursty data streams 
It is important to note that the maximum throughput achieved (around 3 Mbps) stays well below the physical limit of $11 \mathrm{Mbps}$. This is due to the overhead introduced by the Physical and MAC layers. Knowing that CF_POLL_size and $C F \_A C K_{-}$size are both equal to 14 bytes and that $D A T A \_$header_size is equal to 34 bytes according to the standard, substituting the simulation parameters in the formula for the maximum throughput of PCF (see above) we reach the curve depicted in Figure 9

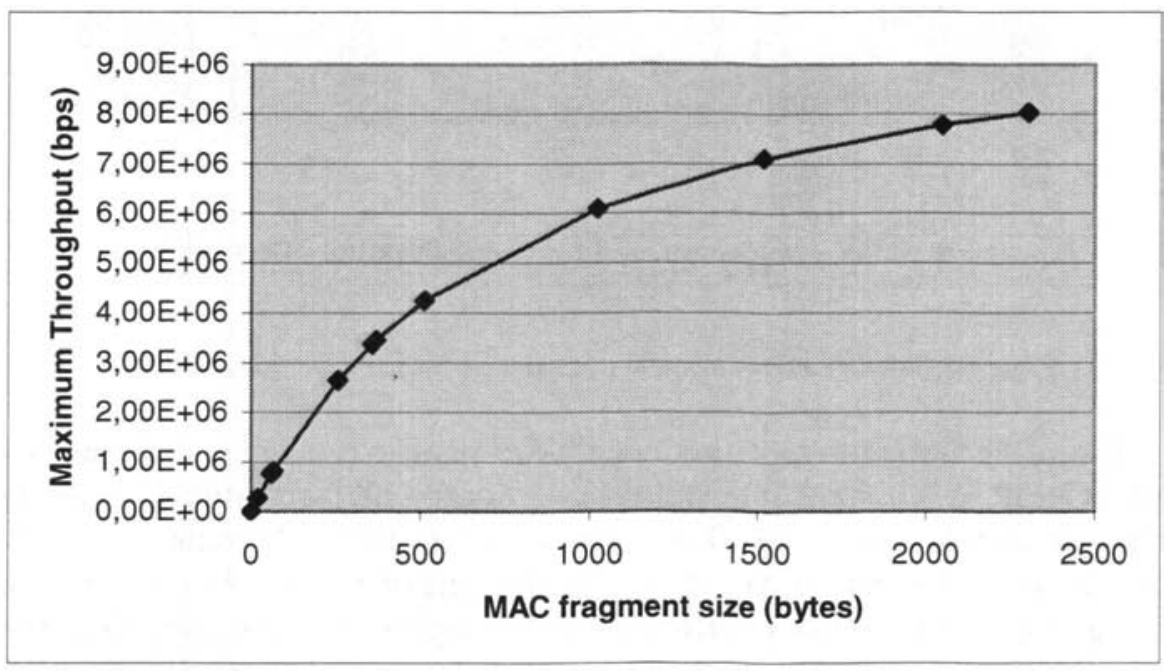

Figure 9. Maximum throughput of $802.11 \mathrm{~b}$ versus MAC fragment size

The average fragment size of the bursty data sources is approximately 357 bytes, as can be easily calculated from the message size distribution. This fragment size corresponds to a maximum throughput of $3.5 \mathrm{Mbps}$, which approximately matches the value achieved for data sources in the simulation. The small difference is explained by the VoP and video sources (a total load of $596 \mathrm{Kbps}$ ) in the simulation and the nonlinearity of the maximum throughput function.

Figure 10 shows the average packet delay for the $\mathrm{VoP}$ and video sessions. As can be seen, SETT is not affected by the increase in the number of data sessions, always presenting delay values around $10 \mathrm{~ms}$. As expected, the WRR service discipline offers no protection to the video and VoP streams, whose delay values start to rise fast for a number of data sessions above 5 and 10 respectively. 


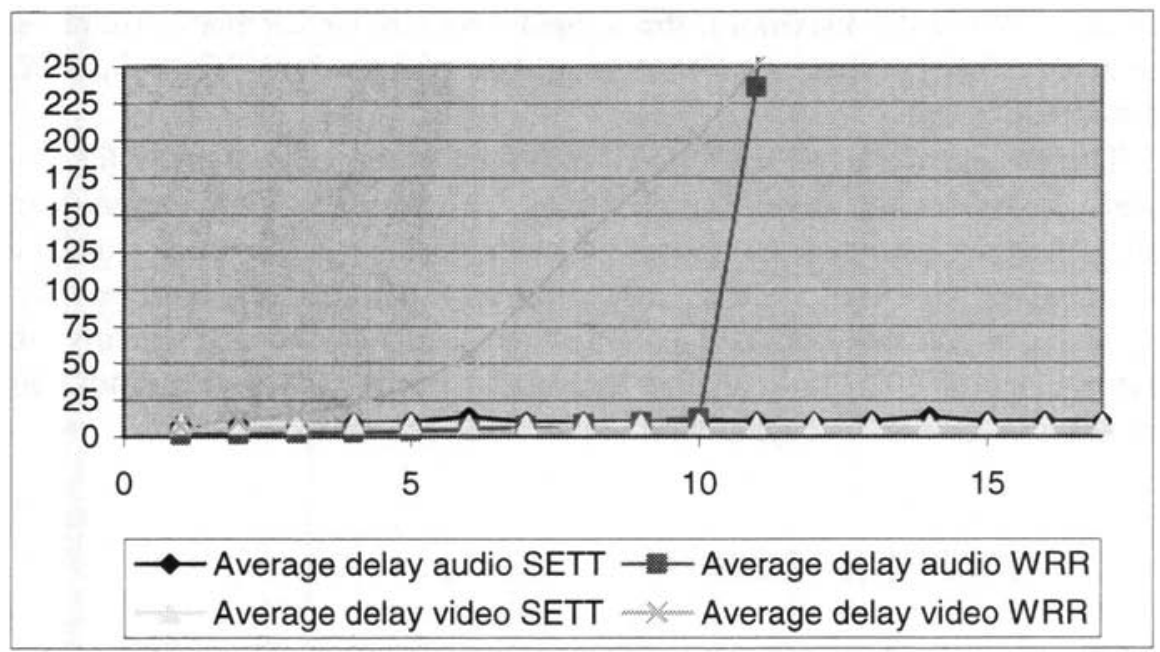

Figure 10. Simulation 2: Average packet delay (ms) for the VoIP and video streams

\section{CONCLUSIONS}

This paper has presented a QoS aware Service Discipline that supports IP QoS in IEEE 802.11 access networks. This service discipline bases its scheduling decisions on the estimated packet transmission times (SETT Scheduling based on Estimated Transmission Times), whose computations are based on the parameters defined in RFC 1363 for FlowSpecs.

SETT was simulated in an $802.11 \mathrm{~b}$ environment and compared with the Weighted Round Robin (WRR) service discipline, considering sources of three different types: bursty data, IP telephony (VoIP) and real-time video. Experiments showed that in general SETT performs better than WRR for VoIP and video streams, especially in presence of bursty data traffic. In this case SETT is able to protect the real-time sessions, keeping their QoS bounds.

The first simulated scenario considered the transmission of one $250 \mathrm{Kbps}$ video session in simultaneous with $n 24 \mathrm{Kbps}$ VoIP sessions. The results show that WRR presents a lower average transmission delay for VoIP traffic, although SETT stays within acceptable values for voice. For more than 17 VoIP sessions, average delay is unacceptable for both disciplines. As for video, SETT performs definitely better than WRR, although in the presence of key frames both disciplines show difficulties to comply with the maximum delay bounds due to the bandwidth limitations of $802.11 \mathrm{~b}$.

The second simulated scenario considered one $250 \mathrm{Kbps}$ video session running simultaneously with two $24 \mathrm{Kbps}$ VoIP sessions and $n$ bursty data 
sessions. While the maximum throughput was similar for both disciplines, the delay of the real-time sessions was greatly affected in WRR, while SETT kept it unaffected.

The present work has left some issues for further study, namely the QoS level achieved when several applications with different QoS requirements run in the same terminal. Another issue concerns the performance of $802.11 \mathrm{~b}$ in a scenario with high mobility, where the PCF periods must be shorter and the physical transmission rate is subject to frequent changes. Finally, the performance of 802.11a is also an interesting subject for further study, and one that can be easily adapted with our simulation tool.

\section{REFERENCES}

[1] IEEE, "Wireless LAN Medium Access Control (MAC) and Physical Layer (PHY) Specifications", IEEE Std. 802.11, June 1997.

[2] J. Weinmiller et al, "Performance Study of Access Control in Wireless LANs - IEEE 802.11 DFWMAC and ETSI RES 10 HIPERLAN", Mobile Networks and Applications (MONET), Volume 2, Number 1, 1997.

[3] J. Weinmiller et al, "Analyzing and Improving the IEEE 802.11-MAC Protocol for Wireless LANs", Proceedings of the 4th Int'l Workshop on Modeling, Analysis and Simulation of Computer and Telecommunication Systems (MASCOTS'96), 1996.

[4] A. Ganz et al, "Robust Superpoll Protocol for IEEE 802.11 Wireless LANs", IEEE Military Communications Conference, Boston, MA, October 1998.

[5] J. Tourrilhes, "PiggyData: Reducing CSMA/CA collisions for multimedia and TCP connections", Proc. of the IEEE Vehicular Technology Conference (VTC'99).

Amsterdam, September 1999.

[6] IETF, "The use of RSVP with the IETF Integrated Services", RFC 2210, September 1997.

[7] IETF, “An Architecture for Differentiated Services", RFC 2475, December 1998.

[8] IETF, “A Proposed Flow Specification", RFC 1363, September 1992.

[9] S. Keshav, An Engineering Approach to Computer Networking. ATM Networks, the Internet, and the Telephone Network; Addison-Wesley Professional Computing Series, Massachusetts, USA, 1997.

[10] IETF, “An Expedited Forwarding PHB”, RFC 2598, June 1999.

[11] M. Macedo, M. Nunes, H. Duarte-Ramos, "SARPVBR - A VBR rate control algorithm for HFC networks", IEEE ATM'97 Workshop, Lisbon, Portugal, May 1997, p. 486-493.

[12] IETF, “Assured Forward PHB Group”, RFC 2597, June 1999.

[13] A. Grilo, M. Nunes, "A Bandwidth Management Algorithm for DPRS”, European Wireless Conference (EW2000). Dresden, September 2000.

[14] IEEE Cable TV Protocol Working Group, "Performance Evaluation Process for MAC Protocols", IEEE 802.34/96-083R3, March 1996.

[15] ITU-T, “One Way Transmission Time”, ITU-T Recommendation G.114, February 1996. 